\title{
Benchmarking Lumbar Puncture Fluoroscopy Time during Fellowship Training
}

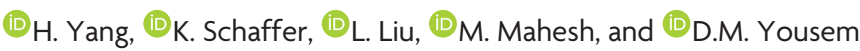

\begin{abstract}
SUMMARY: We sought to establish a guide for identifying fellowship competency in performing fluoroscopically guided lumbar punctures. With a linear mixed-effects model, we compared the fluoroscopy time between the first and last 3 months of neuroradiology training. During 7 years, 55 fellows performed 1142 and 861 lumbar punctures in the first and last quarters of training. A target fluoroscopy time of 0.26 minutes, the upper $95 \%$ confidence interval, can serve as a fellowship benchmark for successfully achieving competence in fluoroscopically guided lumbar punctures.
\end{abstract}

ABBREVIATIONS: FGLP = fluoroscopically guided lumbar puncture; $F T$ = fluoroscopy time

$F$ uoroscopically guided lumbar puncture (FGLP) is often requested in radiology departments when "blind" attempts fail by clinicians. Under the guidance of fluoroscopy, one can visualize real-time bony anatomic landmarks and targets, leading to improved accuracy of needle placement for lumbar puncture in such difficult situations. However, both patients and operators are exposed to ionizing radiation during this procedure. ${ }^{1}$ The risk from radiation exposure accumulates during one's lifetime. ${ }^{2}$ A Sentinel Event Alert published by the Joint Commission in 2011 recommended that one attempt to reduce the radiation dose to "as low as reasonably achievable (ALARA)" without sacrificing patient care. ${ }^{3}$

Both the American Board of Radiology and the Accreditation Council for Graduate Medical Education have listed FGLP as an essential competency for radiology residents and neuroradiology fellows. The American College of Radiology encourages recording the fluoroscopy time (FT) during lumbar punctures and comparing it with benchmarks. ${ }^{4}$ The Radiological Society of North America competency initiative encourages setting parameters for proficiency in procedures. To our knowledge, FT benchmarks have not been established for neuroradiology fellows performing FGLP. We conducted this retrospective study to establish FT

Received September 3, 2016; accepted after revision October 8.

From the Division of Neuroradiology (H.Y., K.S., L.L., M.M., D.M.Y.), The Russell H. Morgan Department of Radiology and Radiological Science, Johns Hopkins Medical Institutions, Baltimore, Maryland; and Shandong Medical Imaging Research Institute (H.Y.), Shandong University, Jinan, Shandong, China.

Please address correspondence to David M. Yousem, MD, MBA, Johns Hopkins Medical Institutions, 600 N. Wolfe St, Phipps B100F, Baltimore, MD 21287;

e-mail: dyousem1@jhu.edu

http://dx.doi.org/10.3174/ajnr.A5031 benchmark data for proficiency assessment during a 1-year neuroradiology fellowship program.

\section{MATERIALS AND METHODS}

This study was performed with institutional review board approval and was Health Insurance Portability and Accountability Act compliant. We retrospectively identified first-year neuroradiology fellows who performed fluoroscopically guided lumbar punctures in the neuroradiology division of Johns Hopkins Medical Institution between 2009 and 2016. All lumbar punctures were performed on an Arcadis Orbic C-Arm fluoroscopic unit (Siemens, Erlangen, Germany) for guidance and were performed in the first year of the neuroradiology fellowship program.

Three lectures ("Radiation Safety," "Spine Procedures," and "Degenerative Spine Disease") on fluoroscopic techniques were given to the first-year fellows in the first 2 months of their fellowship. The fellows were scheduled on weekly rotations for spine procedures, including lumbar punctures and myelography; there were usually 8 first-year fellows per year. This meant that during their first rotation through the spine service, they likely had not completed all 3 educational presentations on lumbar puncture techniques.

Each procedure was monitored in the room by neuroradiology faculty who provided helpful hints and instructions on improving the procedure technique; fellows do not perform the procedures unattended. The fluoroscopy time at the end of the procedure was displayed on the screen of the C-arm system. No spot films were obtained for the lumbar punctures. One senior technologist recorded the fluoroscopy time for each procedure in a logbook. We collected the fluoroscopy time of each lumbar 
puncture in the first 3 months (July, August, and September of the first year of fellowship) and last 3 months of the fellowship (April, May, and June of the same academic year [July-June]) for each neuroradiology fellow. The fluoroscopy times had been recorded for all such procedures from July 2009 to June 2016, the time course for this retrospective study. The fluoroscopy times were not recorded before July 2009. No cervical C1-2 punctures were included.

The fluoroscopy time was analyzed as a continuous variable. The median with interquartile range and mean with $95 \%$ confidence interval were reported. A linear mixed-effects model was used to estimate the average fluoroscopy time at the first and the last quarters of fellowship adjusted for the year of ending the fellowship (2010-2016), the total number of cases performed by each fellow, and within-fellow variation. The distribution of fluoroscopy time was highly skewed; therefore, log-transformed values were used for statistical tests and modeling. The estimated mean and 95\% CI then were converted to the original scale (in minutes) for presentation. Statistical significance was defined at $P<.05$. All analyses were performed by using STATA (StataCorp, College Station, Texas).

\section{RESULTS}

During the 7-year period, 55 neuroradiology fellows performed 1142 lumbar punctures in their first 3 months of fellowship and 861 procedures in their last 3 months of fellowship. Seven fellows did not have data for either the first or the last 3 months of fellowship, and 3 fellows had performed only 1 procedure in 1 timeframe. These 10 fellows were excluded from the comparison anal-

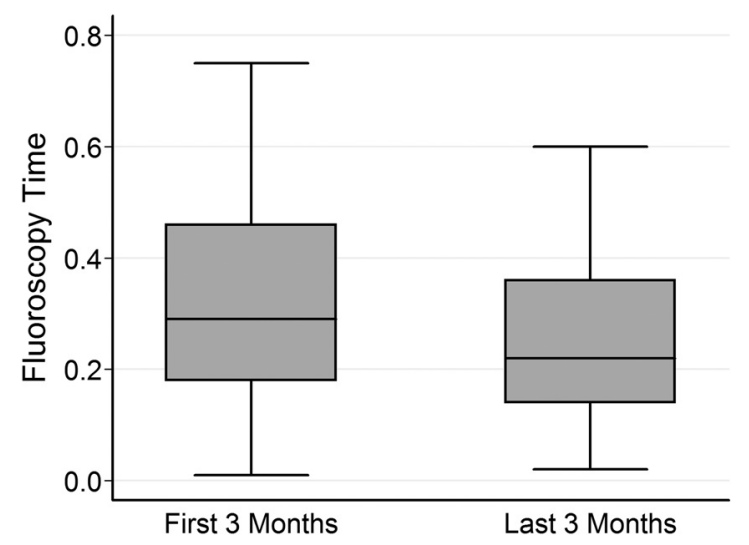

FIG 1. Comparison of fluoroscopy time for FGLP in the first and the last 3 months of the fellowship. yses. The variation between the quarters may reflect the influence of senior residents requesting neuroradiology rotations at the end of the academic year who also had week-long rotations on the spine service (but whose data were not included).

We surveyed the indications for 1 month of the study and found that 49/70 lumbar punctures were performed to instill chemotherapy and withdraw CSF; 8, for suspected high or low intracranial pressure; 5 , to exclude meningitis; 4 , for multiple sclerosis evaluation; 3, for afebrile change in mental status; and 1, for neuropathy.

The average number of procedures performed per fellow in the first and the last 3 months of the fellowship was 19 (median; interquartile range, 14-25) and 18 (median; interquartile range, $10-25)$, respectively. The median of fluoroscopy time was 0.29 minutes (interquartile range, $0.18-0.46$ minutes) for the first 3 months of fellowship and 0.22 minutes (interquartile range, 0.14-0.36 minutes) for the last 3 months of fellowship (Fig 1).

The estimated overall mean fluoroscopy time was $0.31 \mathrm{~min}-$ utes (95\% CI, $0.28-0.33$ minutes) at the beginning of training and 0.24 minutes ( $95 \% \mathrm{CI}, 0.22-0.26$ minutes) at the end of training. There was a $22.1 \%$ reduction in fluoroscopy time when comparing the first and last quarters of the fellowship $(P<.001)$. The total number of cases performed by each fellow was not significantly associated with the fluoroscopy time reduction $(P=.088)$, though the trend showed that the more cases performed, the shorter was the fluoroscopy time. Most (34 of 45, 75.6\%) fellows showed a reduction in fluoroscopy time from the first 3 months to the last 3 months of the fellowship (Table).

\section{DISCUSSION}

This study provides benchmarks for neuroradiology trainees performing FGLP in programs that have similar volumes of cases. We believe that at the end of training, based on data we have from a large number of fellows during several years, a mean target of $<0.26$ minutes ( 16 seconds) of FT should be used to establish that the fellow has gained expertise in that technique. This value for the end of the fellows' practice, on average, represents the upper $95 \%$ confidence interval from our dataset. We acknowledge that on the basis of body habitus and the degree of spinal stenosis, any individual case may take longer or shorter time, but our FT value may be applied to the mean value of several FGLPs performed by a fellow, to assess competency.

Overall, our data show a $22.1 \%$ reduction in fluoroscopy time when comparing the first and last quarters of the fellowship for 7 years, indicating the improvement of the operators' expertise

Average level and reduction of FT in early and later fellowship, adjusting for the total number of cases performed by each fellow

\begin{tabular}{|c|c|c|c|c|c|c|}
\hline Year & $\begin{array}{l}\text { No. of } \\
\text { Fellows }\end{array}$ & $\begin{array}{l}\text { FT in Early Fellowship } \\
\text { (Mean) }(95 \% \mathrm{Cl})(\mathrm{min})\end{array}$ & $\begin{array}{l}\text { FT in Late Fellowship } \\
\text { (Mean) }(95 \% \mathrm{CI})(\mathrm{min})\end{array}$ & Reduction & $\begin{array}{l}\text { Percentage of } \\
\text { Reduction }^{\mathrm{a}}\end{array}$ & $\begin{array}{c}\text { No. of } \\
\text { Improved (\%) }\end{array}$ \\
\hline 2009-10 & 6 & $0.40(0.33-0.49)$ & $0.31(0.26-0.38)$ & 0.09 & 28.8 & $4(66.7)$ \\
\hline 2010-11 & 9 & $0.35(0.29-0.44)$ & $0.27(0.21-0.34)$ & 0.08 & 25.1 & $7(77.8)$ \\
\hline 2011-12 & 5 & $0.27(0.23-0.32)$ & $0.21(0.17-0.25)$ & 0.06 & 19.9 & $3(60.0)$ \\
\hline $2012-13$ & 7 & $0.28(0.23-0.33)$ & $0.21(0.18-0.26)$ & 0.06 & 20.4 & $4(57.1)^{\prime}$ \\
\hline 2013-14 & 6 & $0.39(0.34-0.44)$ & $0.30(0.27-0.34)$ & 0.09 & 27.8 & $6(100.0)$ \\
\hline 2014-15 & 6 & $0.24(0.19-0.29)$ & $0.18(0.15-0.22)$ & 0.05 & 18.1 & $4(66.7)$ \\
\hline 2015-16 & 6 & $0.25(0.21-0.30)$ & $0.19(0.16-0.23)$ & 0.06 & 18.7 & $6(100.0)$ \\
\hline Total & 45 & $0.31(0.28-0.33)$ & $0.24(0.22-0.26)^{b}$ & 0.07 & 22.1 & $34(75.6)$ \\
\hline
\end{tabular}

a The percentage of reduction was based on the log-transformed value.

${ }^{\mathrm{b}}$ There was a significant difference between early and late fellowship $(P<.05)$. 
through training and experience. The data also demonstrate a trend showing that the more cases performed, the shorter was the fluoroscopy time. Our model showed that for every 2 additional cases performed, there was a 0.01 -minute reduction in fluoroscopy time $(P=.088)$. This non-statistically significant association may be caused by a similar number of cases performed by each fellow during the 1-year fellowship.

The initial value of 0.33 minutes ( 20 seconds of FT) at the upper 95\% confidence interval at the beginning of fellowship could represent what one might hope a graduating resident could achieve. The bias herein is that these are residents who are entering neuroradiology fellowships who may have more interest and proficiency in FGLP. This circumstance may explain why the initial FT of our fellows was substantively shorter than that reported by Boddu et $\mathrm{al}^{5}$ for residents and fellows for a normal body mass index (mean, 0.48 minutes; 95\% CI, 0.40-0.56 minutes). Boddu et al noted that the fluoroscopy time of fellows was significantly lower than that of residents $(P=.03)$. This is the only other study that has looked at FT times for FGLP, to our knowledge.

Proper training of radiologists was reported to have effects on the reduction of fluoroscopy time in different procedures. Lim et $\mathrm{al}^{6}$ reported that the fluoroscopy time for voiding cystourethrography of pediatric radiology fellows was shorter than that of senior radiology residents. A statistically significant training effect $(P<.05)$ was demonstrated by Stuart et $\mathrm{al}^{7}$ when performing uterine artery embolization during a 1-year radiology fellowship training. Some programs across the country have begun to emphasize the training of radiology residents in fluoroscopically guided lumbar puncture. A simulation-based fluoroscopically guided lumbar puncture curriculum, including a 1-hour lecture and hands-on training with a lumbar spine phantom, was reported to improve residents' procedure efficiency. ${ }^{8}$ The mean fluoroscopy times for the retrospective resident group and the prospective group were $1.09 \pm$ 0.65 minutes and $0.87 \pm 0.68$ minutes, respectively. These values are approximately 2.5 times higher than those at the beginning and end of fellowship training in our study, respectively.

Fluoroscopy time is one of the most widely reported as a dose metric for fluoroscopic procedures. For interventional procedures, fluoroscopy time alone is not a representative dose descriptor because these procedures often include digital runs (digital subtraction angiography) or spot films; however, for lumbar punctures, there are no spot films or digital runs performed. Thus, fluoroscopy time becomes the key dose metric. A number of dose-optimization strategies ${ }^{9}$ can be applied to reduce the dose to patients and personnel: They include the use of pulse fluoroscopy and collimating the radiation field to the area of interest, and using the last image or last series hold features to avoid unnecessary exposure, especially in a teaching environment. In addition, education and training of fluoroscopists play a role in reducing the radiation dose to patients. Our study demonstrates the impor- tance of training and experience, which leads to reducing the patient dose. For our study purpose, we only compared the fluoroscopy time as representative of the operator's experience.

The study is limited by the exclusion of data points from 10 fellows who did not perform sufficient lumbar punctures in the study periods examined. We do not know the patient characteristics that may influence the fluoroscopy time such as body habitus or significant degenerative changes. We did not record needle lengths or indications as part of our data. We believe the daily FGLP volume of our service is reflective of programs with large fellowships. Of 15 programs that responded to an e-mail survey on the topic, the averages (2.3 FGLPs per day) were slightly lower than our average of 3.8 per day but the range was between zero (CT-directed only) and 5.

\section{CONCLUSIONS}

We established a target mean FT value of 0.26 minutes as one of the criteria of proficiency for fellows for FGLP. Proper training of operators on fluoroscopically guided procedures will reduce the radiation exposure for personnel and patients.

Disclosures: David M. Yousem—UNRELATED: Expert Testimony: self; Payment for Lectures including Service on Speakers Bureaus: ACR Ed Center*; Royalties: Elsevier; Payment for Development of Educational Presentations: CMElnfo.com. * Money paid to the institution.

\section{REFERENCES}

1. Cauley KA. Fluoroscopically guided lumbar puncture. $A J R A m J$ Roentgenol 2015;205:W442-50 CrossRef Medline

2. Amis ES Jr, Butler PF; American College of Radiology. ACR white paper on radiation dose in medicine: three years later. $J$ Am Coll Radiol 2010;7:865-70 CrossRef Medline

3. Radiation risks of diagnostic imaging. Sentinel Event Alert 2011;24: 1-4 Medline

4. Amis ES Jr, Butler PF, Applegate KE, et al; American College of Radiology. American College of Radiology white paper on radiation dose in medicine. J Am Coll Radiol 2007;4:272-84 CrossRef Medline

5. Boddu SR, Corey A, Peterson R, et al. Fluoroscopic-guided lumbar puncture: fluoroscopic time and implications of body mass index-a baseline study. AJNR Am J Neuroradiol 2014;35:1475-80 CrossRef Medline

6. Lim R, Khawaja RD, Nimkin K, et al. Relationship between radiologist training level and fluoroscopy time for voiding cystourethrography. AJR Am J Roentgenol 2013;200:645-51 CrossRef Medline

7. Stuart S, Mayo JR, Ling A, et al. Retrospective study of the impact of fellowship training on two quality and safety measures in uterine artery embolization. J Am Coll Radiol 2014;11:471-76 CrossRef Medline

8. Faulkner AR, Bourgeois AC, Bradley YC, et al. Simulation-based educational curriculum for fluoroscopically guided lumbar puncture improves operator confidence and reduces patient dose. Acad Radiol 2015;22:668-73 CrossRef Medline

9. Mahesh M. Fluoroscopy: patient radiation exposure issues. Radiographics 2001;21:1033-45 CrossRef Medline 